\title{
Toxicidade de inseticidas utilizados na cultura do tomateiro a Trichogramma pretiosum
}

\author{
Alexandre Pinho Moura(1), Geraldo Andrade Carvalho ${ }^{(1)}$ e Renê Luís de Oliveira Rigitano(1) \\ (1)Universidade Federal de Lavras, Dep. de Entomologia, Caixa Postal 3037, CEP 37200-000 Lavras, MG. E-mail: alexandrepm@yahoo.com, \\ gacarval@ufla.br, rigitano@ufla.br
}

Resumo - O objetivo deste trabalho foi avaliar a toxicidade dos inseticidas acetamipride $\left(0,05 \mathrm{~g} \mathrm{~L}^{-1}\right.$ de i.a. $)$, clorfenapir (0,12 $\mathrm{g} \mathrm{L}^{-1}$ de i.a.), imidaclopride (1,16 $\mathrm{g} \mathrm{L}^{-1}$ de i.a.), tiaclopride (0,48 $\mathrm{g} \mathrm{L}^{-1}$ de i.a.) e tiametoxam (0,05 $\mathrm{g} \mathrm{L}^{-1}$ de i.a.) a Trichogramma pretiosum em diferentes fases imaturas. Ovos de Anagasta kuehniella foram aderidos em cartelas de cartolina azul e expostos ao parasitismo de T. pretiosum por 48 horas. Os ovos supostamente parasitados, contendo os parasitóides no período de ovo-larva e nas fases de pré-pupa e pupa, foram imersos nas caldas químicas e em água (testemunha) por cinco segundos, e mantidos em câmara climatizada a $24 \pm 1^{\circ} \mathrm{C}$, UR de $70 \pm 10 \%$ e 12 horas de fotófase. Clorfenapir e imidaclopride reduziram em 76,0\% e 64,4\%, respectivamente, a emergência de indivíduos da geração $\mathrm{F}_{1}$ de $T$. pretiosum. A razão sexual dos indivíduos da geração $\mathrm{F}_{1}$ não foi afetada por nenhum dos compostos avaliados, variando de 0,7 a 0,8 . Clorfenapir reduziu em aproximadamente $50 \%$ a taxa de parasitismo de T. pretiosum da geração $\mathrm{F}_{1}$. Acetamipride e tiametoxam foram inofensivos ao parasitóide e podem ser utilizados em associação com essa espécie no controle de pragas na cultura do tomateiro.

Termos para indexação: parasitóide, tomate, produtos fitossanitários, seletividade.

\section{Toxicity of insecticides used in tomato crop to Trichogramma pretiosum}

\begin{abstract}
The aim of this work was to evaluate the toxicity of the insecticides acetamiprid (0.05 $\mathrm{g} \mathrm{L}^{-1}$ a.i.), chlorfenapyr (0.12 $\mathrm{g} \mathrm{L}^{-1}$ a.i.), imidacloprid (1.16 $\mathrm{g} \mathrm{L}^{-1}$ a.i.), thiacloprid (0.48 $\mathrm{g} \mathrm{L}^{-1}$ a.i.) and thiamethoxam (0.05 $\mathrm{g} \mathrm{L}^{-1}$ a.i.) to Trichogramma pretiosum, in different immature stages. Eggs of Anagasta kuehniella glued in blue paper cards were offered to females of T. pretiosum for 48 hours. After the parasitization, the eggs were treated by dipping the cards into the chemical solutions or in water for five seconds after they reached the egglarval, prepupal and pupal stages. The bioassays were carried out under controlled conditions, at $24 \pm 1^{\circ} \mathrm{C}, \mathrm{RH}$ of $70 \pm 10 \%$ and 12 hours of photophase. Both chlorfenapyr and imidacloprid reduced in $76.0 \%$ and $64.4 \%$, respectively, the emergence of individuals of the $\mathrm{F}_{1}$ generation of $T$. pretiosum. No insecticide affected the sex ratio of the $\mathrm{F}_{1}$ generation of this parasitoid. Chlorfenapyr reduced in approximately $50 \%$ the parasitization rate of individuals of the $\mathrm{F}_{1}$ generation. Both acetamiprid and thiamethoxam were harmless to T. pretiosum, so that they can be used for the control of pests in tomato crop with this parasitoid.
\end{abstract}

Index terms: parasitoid, tomato, pesticides, selectivity.

\section{Introdução}

Parasitóides do gênero Trichogramma têm sido amplamente utilizados em programas de controle biológico de pragas devido à facilidade de sua criação em hospedeiros alternativos (Parra, 1997; Haji et al., 1998b) além de sua agressividade no parasitismo de ovos de insetospraga (Botelho, 1997).

Aproximadamente 18 espécies de Trichogramma vêm sendo criadas massalmente, em cerca de 23 países, para liberações inundativas visando ao controle de pragas das culturas do milho, cana-de-açúcar, tomate, arroz, algodão, soja, beterraba, maçã, ameixa, hortaliças e de reflorestamentos, entre outras (Hassan et al., 1998).

No Brasil, a espécie Trichogramma pretiosum Riley, 1879 (Hymenoptera: Trichogrammatidae) tem sido criada massalmente e liberada em cultivos de tomateiro na região do Submédio São Francisco para o controle da Tuta absoluta Meyrick, 1917 (=Scrobipalpuloides) (Lepidoptera: Gelechiidae), das brocas pequena e grande, Neoleucinodes elegantalis Guenée, 1854 (Lepidoptera: Crambidae) e Helicoverpa zea Boddie, 1850 (=Heliothis) (Lepidoptera: Noctuidae), respecti- 
vamente, bem como para a lagarta das folhas do tomateiro, Manduca diffissa Butler, 1871 (Lepidoptera: Sphingidae), que são fatores limitantes ao desenvolvimento dessa cultura (Haji et al., 1998a).

Entretanto, um dos grandes entraves na utilização desse e de outros parasitóides no controle de pragas do tomateiro é o fato de se continuar utilizando grandes quantidades de produtos químicos para o controle de insetos-praga e doenças nessa cultura. Outro fator limitante é a falta de informação a respeito da ação tóxica de pesticidas mais recentemente lançados no mercado e amplamente utilizados no controle de pragas da tomaticultura, tais como neonicotinóides e pirroles, aos parasitóides que atuam como agentes de controle biológico de pragas nesta cultura.

Os neonicotinóides são registrados para o controle de uma grande variedade de insetos-praga em diversos agroecossistemas. São agonistas do receptor nicotínico da acetilcolina e apresentam elevada capacidade inseticida, principalmente contra lepidópteros-praga, e baixa toxicidade a mamíferos (Yamamoto et al., 1995; Takahashi et al., 1998).

Compostos pertencentes ao grupo dos pirroles, que afetam o metabolismo de energia impedindo a formação de ATP (Treacy et al., 1994) são recomendados para o controle de pragas em diversas culturas, apresentando, também, efeito prejudicial a alguns inimigos naturais, principalmente a parasitóides.

Estudos sobre seletividade de pesticidas a inimigos naturais de pragas, portanto, devem ser realizados com o intuito de gerar informações que possam auxiliar na tomada de decisão em programas de Manejo Integrado de Pragas (MIP) e na manutenção desses organismos nos agroecossistemas, de modo que os mesmos atuem na regulação de populações de pragas.

O objetivo deste trabalho foi avaliar a toxicidade de inseticidas recomendados para a cultura do tomateiro estaqueado sobre o parasitóide de ovos $T$. pretiosum.

\section{Material e Métodos}

Este trabalho foi realizado no período de março a julho de 2002, utilizando-se uma população de $T$. pretiosum proveniente de Piracicaba, SP, obtida de ovos de Spodoptera frugiperda J. E. Smith, 1797 (Lepidoptera: Noctuidae) coletados em cultura de milho.

A traça-da-farinha Anagasta kuehniella Zeller, 1879 (Lepidoptera: Pyralidae) foi utilizada como hospedeiro alternativo para criação de T. pretiosum em laborató- rio. Esse hospedeiro foi criado de acordo com a metodologia descrita por Parra (1997), utilizando-se dieta artificial composta de farinha de trigo (97\%) e lêvedo de cerveja (3\%). Ovos do hospedeiro foram aderidos a cartelas de cartolina azul com $8 \mathrm{~cm}$ de comprimento e $2 \mathrm{~cm}$ de largura, utilizando-se goma arábica diluída a $50 \%$ em água. Esses ovos foram, posteriormente, submetidos à inviabilização sob lâmpada germicida, conforme descrito por Stein \& Parra (1987), sendo posteriormente expostos ao parasitismo por um período de 24 horas e mantidos em câmara climatizada a $24 \pm 1{ }^{\circ} \mathrm{C}$, UR de $70 \pm 10 \%$ e fotófase de 12 horas, até a emergência dos parasitóides, os quais receberam novos ovos, dando início a outro ciclo de desenvolvimento. Utilizaram-se apenas parasitóides a partir da terceira geração de laboratório e com até 12 horas de idade, para a realização dos bioensaios.

Os inseticidas utilizados nos testes com T. pretiosum foram acetamipride $\left(0,05 \mathrm{~g} \mathrm{~L}^{-1}\right.$ de i.a. $)$, clorfenapir $\left(0,12 \mathrm{~g} \mathrm{~L}^{-1}\right.$ de i.a.), imidaclopride $\left(1,16 \mathrm{gL}^{-1}\right.$ de i.a.), tiaclopride $\left(0,48 \mathrm{~g} \mathrm{~L}^{-1}\right.$ de i.a. $)$ e tiametoxam $\left(0,05 \mathrm{~g} \mathrm{~L}^{-1}\right.$ dei.a. $)$. As dosagens utilizadas correspondem às mais elevadas recomendadas pelos fabricantes para o controle de pragas na cultura do tomateiro. Cada produto foi considerado um tratamento, sendo um sexto tratamento inserido como testemunha, em que foi utilizada apenas água destilada.

Em cada tratamento, quarenta fêmeas com idade de até 12 horas foram individualizadas em tubos de vidro de $8 \mathrm{~cm}$ de altura $x 2,5 \mathrm{~cm}$ de diâmetro, fechados com filme de polietileno, sendo alimentadas com mel em forma de gotículas depositadas no interior dos recipientes. Cerca de 125 ovos de A. kuehniella com até 24 horas de idade foram aderidos a cartelas de cartolina azul com $5 \mathrm{~cm}$ de comprimento e $0,5 \mathrm{~cm}$ de largura, por meio de goma arábica diluída a 50\% em água, inviabilizados sob lâmpada germicida e ofertados aos parasitóides. Após 48 horas, as fêmeas foram descartadas e os ovos supostamente parasitados foram mantidos em câmara climatizada sob as mesmas condições da criação, até os parasitóides atingirem cada estágio de desenvolvimento desejado para a realização dos bioensaios.

Quarenta cartelas com ovos de A. kuehniella, por tratamento, contendo o parasitóide no período de ovolarva ou nas fases de pré-pupa ou pupa $(0-48 \mathrm{~h}$, 72-120 h, 168-216 h, respectivamente), em um total de 240 cartelas para cada estágio de desenvolvimento, foram imersas nas caldas químicas e em água (testemunha) por 5 segundos e, em seguida, colocadas em novos tubos que foram mantidos em câmara climatizada nas 
mesmas condições descritas anteriormente. Cada tratamento foi composto por dez repetições, sendo cada parcela constituída de quatro cartelas com ovos de A. kuehniella contendo o parasitóide em suas diferentes fases imaturas, totalizando 40 cartelas por tratamento.

Avaliaram-se os efeitos dos inseticidas sobre os adultos recém-emergidos dos ovos tratados durante os diferentes estágios imaturos desse parasitóide. Assim, vinte fêmeas de $T$. pretiosum da geração $\mathrm{F}_{1}$, por tratamento, foram individualizadas em tubos de vidro fechados com filme de polietileno e alimentadas com gotículas de mel depositadas na parede do recipiente. A essas fêmeas ofertaram-se cerca de 125 ovos de A. kuehniella com até 24 horas de idade, não tratados, os quais foram previamente aderidos em cartela de cartolina azul e inviabilizados sob lâmpada germicida. O período de parasitismo foi de 48 horas, findo o qual, as fêmeas foram descartadas e as cartelas contendo os ovos supostamente parasitados foram mantidas em câmara climatizada nas condições citadas, até o completo desenvolvimento do parasitóide. Cada tratamento foi composto por dez repetições, sendo cada parcela formada de duas cartelas contendo ovos do hospedeiro.

A toxicidade dos produtos para cada fase de desenvolvimento do parasitóide foi mensurada por meio da avaliação da taxa de parasitismo (número de ovos parasitados/fêmea da geração $F_{1}$ ), porcentagem de emergência (número de ovos com orifício de saída do parasitóide/número total de ovos parasitados), razão sexual (número de fêmeas/número de fêmeas + número de machos) e porcentagem de deformação (número de indivíduos deformados/número total de indivíduos).

Foi utilizado delineamento experimental inteiramente ao acaso, em esquema fatorial $3 \times 6$, três períodos de desenvolvimento $\mathrm{x}$ seis tratamentos. Os dados foram submetidos à análise de variância e as médias comparadas pelo teste de Scott-Knott (Scott \& Knott, 1974).

Os inseticidas também foram enquadrados em classes toxicológicas, de acordo com a redução da capacidade benéfica do parasitóide em relação ao tratamento testemunha, conforme escala proposta por membros da "International Organization for Biological and Integrated Control of Noxious Animals and Plants (IOBC)" (Sterk et al., 1999), em que a classe 1 compreende os inseticidas inofensivos ( $<30 \%$ de redução), a classe 2 , os pouco prejudiciais ( $30 \%$ a $79 \%$ de redução), a classe 3 , os moderadamente prejudiciais ( $80 \%$ a $99 \%$ de redução) e a classe 4 , os prejudiciais (>99\% de redução). A porcentagem média de redução da capacidade benéfica do parasitóide (parasitismo e emergência) foi obtida por meio da equação: \% redução $=100-[$ (média geral do tratamento/média geral da testemunha) x 100].

\section{Resultados e Discussão}

Clorfenapir foi altamente tóxico a T. pretiosum, reduzindo a taxa de emergência de indivíduos da geração $\mathrm{F}_{1}$, quando aplicado sobre ovos de A. kuehniella contendo o parasitóide no período de ovo-larva, com emergência de apenas 19,4\%. Acetamipride, imidaclopride e tiaclopride apresentaram efeito intermediário, diminuindo significativamente a emergência de indivíduos da geração $\mathrm{F}_{1}$ de $T$. pretiosum, com médias de $80 \%, 55 \%$ e $87 \%$, respectivamente. Apenas tiametoxam, dentre os compostos avaliados, não afetou negativamente a taxa de emergência (Tabela 1).

Em estudos desenvolvidos por Schuld \& Schmuck (1997) não se constataram efeitos do imidaclopride quando pulverizado nas dosagens de 0,15 e $0,175 \mathrm{gL}^{-1}$ de i.a. sobre ovos de Cydia pomonella Linnaeus, 1758 (Lepidoptera: Tortricidae) parasitados, contendo os estágios imaturos de Trichogramma cacoeciae Marchal, 1927. Essa divergência de resultados pode estar associada à maior dosagem do imidaclopride utilizada ou às diferentes técnicas de tratamento dos ovos do hospedeiro com o inseticida. Variações interespecíficas na sensibilidade do parasitóide ou do hospedeiro ao imidaclopride também podem, ao menos em parte, ter sido a causa da divergência dos resultados, uma vez que os neonicotinóides apresentam toxicidade bastante variável de acordo com o grupo ou espécie de inseto (Takahashi et al., 1998).

No tratamento de ovos do hospedeiro contendo parasitóides na fase de pré-pupa, todos os compostos avaliados reduziram significativamente a taxa de emergência dos indivíduos da geração $F_{1}$, sendo clorfenapir e imidaclopride os mais prejudiciais, com médias de 2,4\% e $18,6 \%$, respectivamente. Os demais produtos permitiram emergência entre $65,8 \%$ e $68,1 \%$ (Tabela 1 ).

Clorfenapir, imidaclopride e tiaclopride foram os compostos mais tóxicos quando aplicados sobre ovos de A. kuehniella contendo $T$. pretiosum na fase de pupa, provocando emergência média de apenas 37,3\%, 13,9\% e $55,4 \%$ dos indivíduos da geração $\mathrm{F}_{1}$, respectivamente (Tabela 1). Os resultados obtidos para tiaclopride dife- 
renciam-se daqueles de Schuld \& Schmuck (2000), que não observaram redução significativa na emergência de $T$. cacoeciae quando ovos de $C$. pomonella contendo seus estágios imaturos foram pulverizados com esse inseticida na dosagem de $0,12 \mathrm{gL}^{-1}$ de i.a. Essas divergências podem estar relacionadas às dosagens utilizadas desse composto, que nos estudos de Schuld \& Schmuck (2000) correspondeu a um quarto da dosagem utilizada neste trabalho, bem como à utilização de diferentes espécies de Trichogramma e de hospedeiro, e de variações nas técnicas utilizadas na aplicação dos produtos.

No período de ovo-larva, T. pretiosum mostrou-se mais tolerante, enquanto as fases de pré-pupa e de pupa desse parasitóide foram as mais afetadas pelos inseticidas avaliados, apresentando emergência média de 49,4\% e 56,4\%, respectivamente (Tabela 1). Entretanto, Carvalho et al. $(2001,2003)$ não constataram diferenças na suscetibilidade dos estágios de desenvolvimento de T. pretiosum quando aplicaram alguns pesticidas recomendados para o controle de pragas do tomateiro sobre ovos de A. kuehniella, contendo os vários estágios imaturos desse parasitóide. Em virtude das variações observadas na tolerância desse organismo aos pesticidas avaliados, relacionadas aos diversos estágios de desenvolvimento de $T$. pretiosum, a escolha da melhor época de aplicação desses produtos químicos deve ser levada em consideração, de modo a minimizar o impacto desses compostos ao parasitóide.

Em função da diminuição da taxa de emergência de $T$. pretiosum, acetamipride, tiaclopride e tiametoxam foram enquadrados na classe $1-$ inofensivos $(<30 \%$ de redução) -, ao passo que clorfenapir e imidaclopride foram classificados como pouco prejudiciais - classe 2 (30\% a $79 \%$ de redução) (Tabela 1). A redução na emergência do parasitóide causada por esses insetici- das ocorreu pela ingestão de seus resíduos quando da abertura do orifício de emergência, conforme observações de Cônsoli et al. (2001), ou mesmo ainda dentro do ovo hospedeiro, devido à capacidade de alguns produtos atravessarem o córion, como foi discutido por Guifen \& Hirai (1997) e Schuld \& Schmuck (2000).

Acetamipride e imidaclopride causaram maior número de deformação quando aplicados sobre ovos do hospedeiro contendo os parasitóides no período de ovo-larva, não sendo prejudiciais para os demais estágios de desenvolvimento dos indivíduos da geração $F_{1}$. Apenas tiametoxam, entre os compostos avaliados, apresentou aumento significativo no número de adultos de T. pretiosum deformados, quando aplicado sobre ovos hospedeiros contendo a fase de pré-pupa desse parasitóide. Todos os produtos, quando aplicados sobre o parasitóide na fase de pupa, não provocaram aumentos significativos do número de indivíduos deformados. Em geral, o período de ovo-larva de T. pretiosum mostrou-se o mais suscetível à indução de deformações causada pelos compostos avaliados (Tabela 1), provavelmente devido à maior atividade das larvas, expondoas ao maior contato com as substâncias que penetraram o ovo, como discutido por Carvalho et al. (2001).

A taxa de parasitismo de T. pretiosum $\left(\mathrm{F}_{1}\right)$ proveniente de ovos do hospedeiro tratados, contendo os parasitóides no período de ovo-larva, não foi afetada pelos inseticidas acetamipride, imidaclopride, tiaclopride e tiametoxam, com médias de 40,5, 34,2, 40,5 e 45,7 ovos parasitados por fêmea, respectivamente. Apenas clorfenapir diferiu significativamente dos demais tratamentos, com média de 15,6 ovos parasitados por fêmea (Tabela 2). Esse mesmo produto também reduziu o número de ovos parasitados por T. pretiosum $\left(\mathrm{F}_{1}\right)$, emergidos de ovos de A. kuehniella tratados quando os indivíduos encontravam-se na fase

Tabela 1. Porcentagem $( \pm \mathrm{EP})$ de emergência e de adultos deformados de Trichogramma pretiosum $\left(\mathrm{F}_{1}\right)$ provenientes de ovos de Anagasta kuehniella tratados, contendo o parasitóide nas diferentes fases imaturas ${ }^{(1)}$.

\begin{tabular}{|c|c|c|c|c|c|c|c|c|c|}
\hline \multirow[t]{2}{*}{ Tratamento } & \multicolumn{6}{|c|}{ Emergência } & \multicolumn{3}{|c|}{ Adultos deformados } \\
\hline & Ovo-larva & Pré-pupa & Pupa & Média & Redução $(\%)^{(2)}$ & Classe $^{(3)}$ & Ovo-larva & Pré-pupa & Pupa \\
\hline Acetamipride & $80,4 \pm 2,16 \mathrm{aC}$ & $65,8 \pm 2,09 \mathrm{cB}$ & $73,0 \pm 1,52 \mathrm{bB}$ & 73,1 & 10,9 & 1 & $6,4 \pm 1,64 \mathrm{aB}$ & $0,7 \pm 0,32 \mathrm{bA}$ & $1,8 \pm 0,89 \mathrm{bA}$ \\
\hline Clorfenapir & $19,4 \pm 2,43 \mathrm{bE}$ & $2,4 \pm 0,79 \mathrm{cD}$ & $37,3 \pm 4,71 \mathrm{aD}$ & 19,7 & 76,0 & 2 & $4,5 \pm 1,59 \mathrm{aA}$ & $0,0 \pm 0,00 \mathrm{bA}$ & $1,7 \pm 0,68 \mathrm{bA}$ \\
\hline Imidaclopride & $55,2 \pm 3,96 \mathrm{aD}$ & $18,6 \pm 2,32 \mathrm{bC}$ & $13,9 \pm 1,93 \mathrm{bE}$ & 29,2 & 64,4 & 2 & $8,6 \pm 1,84 \mathrm{aB}$ & $0,5 \pm 0,30 \mathrm{cA}$ & $4,1 \pm 1,75 \mathrm{bA}$ \\
\hline Tiaclopride & $87,1 \pm 1,41 \mathrm{aB}$ & $66,1 \pm 2,85 \mathrm{bB}$ & $55,4 \pm 3,43 \mathrm{cC}$ & 69,5 & 15,2 & 1 & $4,7 \pm 0,73 \mathrm{aA}$ & $2,3 \pm 0,76 \mathrm{aA}$ & $3,2 \pm 1,08 \mathrm{aA}$ \\
\hline Tiametoxam & $92,4 \pm 1,17 \mathrm{aA}$ & $68,1 \pm 1,65 \mathrm{cB}$ & $83,5 \pm 1,62 \mathrm{bA}$ & 81,3 & 0,9 & 1 & $4,5 \pm 1,25 \mathrm{aA}$ & $6,0 \pm 1,08 \mathrm{aB}$ & $2,0 \pm 0,76 \mathrm{bA}$ \\
\hline Testemunha & $95,3 \pm 0,74 \mathrm{aA}$ & $75,6 \pm 1,38 \mathrm{bA}$ & $75,0 \pm 2,54 \mathrm{bB}$ & 82,0 & - & - & $2,3 \pm 0,85 \mathrm{aA}$ & $1,6 \pm 0,33 \mathrm{aA}$ & $0,0 \pm 0,00 \mathrm{aA}$ \\
\hline Média & 71,6 & 49,4 & 56,4 & & & & 5,2 & 1,9 & 2,1 \\
\hline
\end{tabular}

${ }^{(1)}$ Médias seguidas pela mesma letra, minúscula na linha e maiúscula na coluna, não diferem entre si pelo teste de Scott-Knott (P<0,05); dados transformados para o arc sen da raiz quadrada de x/100; CV (\%): 10,3 (emergência) e 60,4 (adultos deformados). (2)Porcentagem média de redução na capacidade de emergência em comparação ao tratamento testemunha. ${ }^{(3)}$ Classe de toxicidade recomendada por Sterk et al. (1999); classe 1: inofensivo; classe 2: pouco prejudicial. 
de pré-pupa (13,5 ovos parasitados/fêmea), não diferindo significativamente do período de ovo-larva. Os tratamentos testemunha e tiametoxam, quando aplicados sobre ovos do hospedeiro contendo a fase de prépupa desse parasitóide, não diferiram entre si, com médias de 37,2 e 33,3 ovos parasitados por fêmea, respectivamente. Durante essa mesma fase, acetamipride, imidaclopride e tiaclopride não afetaram a taxa de parasitismo dos parasitóides da geração $\mathrm{F}_{1}$, com médias de 44,1, 40,5 e 42,4 ovos parasitados por fêmea, respectivamente (Tabela 2).

No estágio de pupa, apenas clorfenapir e imidaclopride reduziram significativamente a taxa de parasitismo de fêmeas da geração $F_{1}$, permitindo parasitismo médio de 27,3 e 33,3 ovos por fêmea, respectivamente. No entanto, o número médio de ovos parasitados por fêmeas provenientes de hospedeiros tratados com imidaclopride durante o período de ovo-larva e as fases de pré-pupa e pupa não diferiu significativamente entre si. Quanto a clorfenapir, houve diferença significativa entre a fase de pupa e os demais estágios de desenvolvimento desse parasitóide. Acetamipride, tiaclopride e tiametoxam não afetaram a taxa de parasitismo para os indivíduos da $\mathrm{F}_{1}$, com médias variando de 40,5 a 45,1 ovos parasitados por fêmea (Tabela 2).

As reduções na taxa de parasitismo causadas pelo clorfenapir indicam um efeito residual desse inseticida sobre os indivíduos da geração $F_{1}$. Nesse caso, a concentração do composto nos indivíduos teria resultado em efeito letal, diminuindo sua longevidade, ou mesmo subletal, reduzindo sua capacidade de parasitismo, ambos os efeitos causando redução na taxa de parasitismo desses indivíduos. Imidaclopride surtiu esse mesmo efeito, porém sobre fêmeas da geração $F_{1}$ que mantiveram contato com resíduos desse composto na superfície do córion no momento de sua emergência, quando aplicado sobre ovos do hospedeiro contendo T. pretiosum apenas na fase de pupa.

As reduções observadas na porcentagem de emergência e no número de ovos parasitados de $T$. pretiosum $\left(\mathrm{F}_{1}\right)$, provenientes de ovos tratados com clorfenapir, independentemente do período em que esse produto foi aplicado (Tabelas 1 e 2), podem estar associadas ao prolongado efeito residual desse produto, já que em observações realizadas por McLeod et al. (2002), na cultura da berinjela, esse composto apresentou persistência de aproximadamente dez dias. Imidaclopride apresentou essa mesma característica, quando os ovos do hospedeiro contendo parasitóides na fase de pupa foram tratados com esse produto (Tabela 2).

Em função da redução na taxa de parasitismo de fêmeas $\left(F_{1}\right)$ causada pelos inseticidas avaliados, acetamipride, imidaclopride, tiaclopride e tiametoxam foram enquadrados na classe $1-$ inofensivos $(<30 \%$ de redução) -, ao passo que clorfenapir foi categorizado como pouco prejudicial - classe 2 (30\% a $79 \%$ de redução) (Tabela 2).

Indivíduos da geração $\mathrm{F}_{1}$ oriundos de ovos do hospedeiro tratados durante as fases imaturas do parasitóide, não tiveram a razão sexual afetada negativamente pelos inseticidas avaliados. O mesmo foi observado quando se avaliou a razão sexual em função dos produtos aplicados (Tabela 3). Com imidaclopride ( $0,28 \mathrm{~g} \mathrm{~L}^{-1}$ de i.a.), Carvalho et al. (2003) observaram menor proporção de fêmeas para a geração $\mathrm{F}_{1}$ de T. pretiosum oriundos de Sete Lagoas, MG, parasitando ovos de A. kuehniella, e obtiveram razão sexual média de $0,55,0,54$ e 0,55 no período de ovolarva e nas fases de pré-pupa e pupa, respectivamente. Essas diferenças de respostas biológicas podem estar associadas às distintas populações de $T$. pretiosum es-

Tabela 2. Número de ovos parasitados $( \pm \mathrm{EP})$ por fêmea de Trichogramma pretiosum da geração $\mathrm{F}_{1}$ oriunda de ovos de Anagasta kuehniella tratados, contendo o parasitóide nas diferentes fases imaturas ${ }^{(1)}$.

\begin{tabular}{lcccccc}
\hline Tratamento & Ovo-larva & Pré-pupa & Pupa & Média & Redução (\%) $^{(2)}$ & Classe $^{(3)}$ \\
\hline Acetamipride & $40,5 \pm 1,27 \mathrm{aA}$ & $44,1 \pm 1,49 \mathrm{aA}$ & $40,5 \pm 2,69 \mathrm{aA}$ & 41,7 & 0,0 & 1 \\
Clorfenapir & $15,6 \pm 2,77 \mathrm{bB}$ & $13,5 \pm 1,56 \mathrm{bC}$ & $27,3 \pm 4,61 \mathrm{aB}$ & 18,8 & 50,5 & 2 \\
Imidaclopride & $34,2 \pm 4,25 \mathrm{aA}$ & $40,5 \pm 2,16 \mathrm{aA}$ & $33,3 \pm 3,74 \mathrm{aB}$ & 36,0 & 5,3 & 1 \\
Tiaclopride & $40,5 \pm 1,24 \mathrm{aA}$ & $42,4 \pm 1,59 \mathrm{aA}$ & $45,1 \pm 1,85 \mathrm{aA}$ & 42,7 & 0,0 & 1 \\
Tiametoxam & $45,7 \pm 3,50 \mathrm{aA}$ & $33,3 \pm 1,51 \mathrm{bB}$ & $41,3 \pm 2,67 \mathrm{aA}$ & 40,1 & 0,0 & 1 \\
Testemunha & $38,6 \pm 2,67 \mathrm{aA}$ & $37,2 \pm 2,30 \mathrm{aB}$ & $38,1 \pm 2,33 \mathrm{aA}$ & 38,0 & - & - \\
\hline Média & 35,9 & 35,2 & 37,6 & & & \\
\hline
\end{tabular}

${ }^{(1)}$ Médias seguidas pela mesma letra, minúscula na linha e maiúscula na coluna, não diferem entre si pelo teste de Scott-Knott $(\mathrm{P}<0,05)$; CV $(\%)$ : 22,5. (2)Porcentagem média de redução na capacidade de parasitismo em comparação ao tratamento testemunha. ${ }^{\left({ }^{3}\right)}$ Classe de toxicidade recomendada por Sterk et al. (1999); classe 1: inofensivo; classe 2: pouco prejudicial. 
tudadas, bem como às suas origens geográficas, como também discutido por Bleicher \& Parra (1990) e Brunner et al. (2001).

Diferenças significativas foram observadas em relação à razão sexual de indivíduos da geração $F_{2}$ desse parasitóide. No período de ovo-larva, clorfenapir diferiu significativamente dos demais tratamentos, apresentando razão sexual de 0,2. Clorfenapir e imidaclopride reduziram significativamente a razão sexual de indivíduos da geração $\mathrm{F}_{2}$, quando aplicados durante a fase de prépupa do parasitóide, ocorrendo exclusivamente a emergência de indivíduos machos. Os inseticidas clorfenapir e imidaclopride, quando aplicados durante a fase de pupa, provocaram razão sexual média de 0,2 e 0,1 , respectivamente. Acetamipride não afetou a razão sexual desse parasitóide, independentemente da fase de desenvolvimento em que foi aplicado, com média de 0,6, 0,7 e 0,6 no período de ovo-larva e nas fases de pré-pupa e pupa, respectivamente. O mesmo ocorreu quando da aplicação de tiametoxam, em que a razão sexual média observada foi de $0,7,0,7$ e 0,7 , respectivamente, nas fases citadas (Tabela 3 ).

Alterações na razão sexual de inimigos naturais expostos a inseticidas também foram observadas por outros autores. Novozhilov et al. (1973) verificaram que

Tabela 3. Razão sexual ( \pm EP) de indivíduos das gerações $F_{1}$ e $\mathrm{F}_{2}$ de Trichogramma pretiosum oriundos de ovos de Anagasta kuehniella tratados, contendo o parasitóide nas diferentes fases imaturas ${ }^{(1)}$.

\begin{tabular}{|c|c|c|c|}
\hline Tratamento & Ovo-larva & Pré-pupa & Pupa \\
\hline & & $\mathrm{F}_{1}$ & \\
\hline Acetamipride & $0,7 \pm 0,03 \mathrm{aA}$ & $0,7 \pm 0,04 \mathrm{aA}$ & $0,7 \pm 0,02 \mathrm{aA}$ \\
\hline Clorfenapir & $0,7 \pm 0,05 \mathrm{aA}$ & $0,7 \pm 0,14 \mathrm{aA}$ & $0,7 \pm 0,03 \mathrm{aA}$ \\
\hline Imidaclopride & $0,7 \pm 0,02 \mathrm{aA}$ & $0,7 \pm 0,04 \mathrm{aA}$ & $0,8 \pm 0,05 \mathrm{aA}$ \\
\hline Tiaclopride & $0,7 \pm 0,02 \mathrm{aA}$ & $0,7 \pm 0,03 \mathrm{aA}$ & $0,7 \pm 0,03 \mathrm{aA}$ \\
\hline Tiametoxam & $0,7 \pm 0,02 \mathrm{aA}$ & $0,7 \pm 0,01 \mathrm{aA}$ & $0,7 \pm 0,03 \mathrm{aA}$ \\
\hline Testemunha & $0,7 \pm 0,04 \mathrm{aA}$ & $0,7 \pm 0,02 \mathrm{aA}$ & $0,7 \pm 0,02 \mathrm{aA}$ \\
\hline \multirow[t]{2}{*}{ Média } & 0,7 & 0,7 & 0,7 \\
\hline & & $\mathrm{F}_{2}$ & \\
\hline Acetamipride & $0,6 \pm 0,03 \mathrm{aA}$ & $0,7 \pm 0,01 \mathrm{aA}$ & $0,6 \pm 0,04 \mathrm{aA}$ \\
\hline Clorfenapir & $0,2 \pm 0,09 \mathrm{aB}$ & $0,0 \pm 0,00 \mathrm{bC}$ & $0,2 \pm 0,04 \mathrm{aC}$ \\
\hline Imidaclopride & $0,6 \pm 0,08 \mathrm{aA}$ & $0,0 \pm 0,00 \mathrm{bC}$ & $0,1 \pm 0,08 \mathrm{bC}$ \\
\hline Tiaclopride & $0,7 \pm 0,04 \mathrm{aA}$ & $0,4 \pm 0,04 \mathrm{bB}$ & $0,4 \pm 0,07 \mathrm{bB}$ \\
\hline Tiametoxam & $0,7 \pm 0,03 \mathrm{aA}$ & $0,7 \pm 0,02 \mathrm{aA}$ & $0,7 \pm 0,01 \mathrm{aA}$ \\
\hline Testemunha & $0,7 \pm 0,02 \mathrm{aA}$ & $0,5 \pm 0,13 \mathrm{aB}$ & $0,5 \pm 0,18 \mathrm{aB}$ \\
\hline Média & 0,6 & 0,4 & 0,4 \\
\hline
\end{tabular}

Trissolcus grandis Thomson, 1861 (Hymenoptera: Scelionidae), quando exposto a triclorfon ainda no interior dos ovos de seu hospedeiro, produziu menor proporção de fêmeas na geração seguinte, quando comparado ao tratamento controle. Efeito oposto foi demonstrado por O'Brien et al. (1985), quando descendentes adultos de Bracon mellitor Say, 1836 (Hymenoptera: Braconidae), tratados com a $\mathrm{LC}_{5}$ de azinfós-metil e clordimeforme, mostraram uma maior relação fêmea:macho em comparação aos descendentes não tratados. Essas alterações observadas podem ser resultantes do que Croft (1990) denominou de efeitos latentes, que se expressam no estágio de vida subseqüente àquele inicialmente exposto ao pesticida. No entanto, as bases fisiológicas de tais efeitos ainda não foram bem esclarecidas.

A porcentagem de emergência de indivíduos da geração $\mathrm{F}_{2}$ de $T$. pretiosum, provenientes de ovos de A. kuehniella contendo parasitóides no período de ovolarva não foi afetada por nenhum dos inseticidas avaliados (Tabela 4). Resultados diferentes foram observados por Carvalho et al. (2003) quanto ao imidaclopride, que registraram emergência de $T$. pretiosum de apenas $68,4 \%$ na geração $F_{2}$. As diferenças de respostas biológicas em função dos produtos podem estar associadas às distintas populações de $T$. pretiosum estudadas, bem como à origem geográfica das mesmas (Bleicher \& Parra, 1990; Brunner et al., 2001). Carvalho et al. (2001, 2004) sugeriram que uma maior exposição de populações de insetos a determinado inseticida, ocorrendo em seu hábitat de origem, pode provocar a seleção de indivíduos mais tolerantes, diferenciando as respostas das populações em condições de laboratório a substâncias químicas.

$\mathrm{Na}$ fase de pré-pupa, nenhum dos compostos avaliados reduziu significativamente a emergência de indivíduos da geração $\mathrm{F}_{2}$ de $T$. pretiosum, em comparação ao tratamento testemunha. No entanto, a porcentagem de emergência de indivíduos oriundos de ovos tratados com clorfenapir, imidaclopride e tiaclopride foi significativamente superior à da testemunha, com médias de 96,3\%, 97,5\% e 95,0\%, respectivamente (Tabela 4).

Indivíduos da geração $\mathrm{F}_{2}$ oriundos de ovos do hospedeiro submetidos a todos os tratamentos durante a fase de pupa do parasitóide, apresentaram porcentagem de emergência entre $92,6 \%$ e 96,4\% (Tabela 4). 
Os resultados observados para o imidaclopride, nas fases de pré-pupa e pupa, assemelham-se aos obtidos por Carvalho et al. (2003), que obtiveram emergência média de $92,1 \%$ e $79,6 \%$ para $T$. pretiosum da geração $F_{2}$ nas fases de pré-pupa e pupa, respectivamente, parasitando ovos da traça-da-farinha A. kuehniella.

Entre os diferentes estágios de desenvolvimento do parasitóide, não foram observadas variações negativas em relação à porcentagem de emergência de indivíduos provenientes de ovos tratados com os inseticidas avaliados (Tabela 4), evidenciando a falta de ocorrência do que Croft (1990) denominou de efeitos latentes, ou seja, os que se expressam no estágio de vida subseqüente àquele exposto inicialmente ao pesticida.

Na geração $\mathrm{F}_{2}$ de $T$. pretiosum, não foram observadas diferenças significativas em relação à porcentagem de deformação de indivíduos provenientes de ovos de A. kuehniella tratados, contendo os parasitóides no período de ovo-larva e na fase de pupa. Na fase de prépupa, a aplicação de acetamipride, clorfenapir, imidaclopride e tiaclopride resultou em porcentagem média de adultos deformados entre $0,0 \%$ e $0,6 \%$, diferindo significativamente do tratamento testemunha e do tiametoxam, que apresentaram médias de $1,4 \%$ e $0,9 \%$,

Tabela 4. Porcentagem ( \pm EP) de emergência e de adultos deformados de Trichogramma pretiosum $\left(\mathrm{F}_{2}\right)$, provenientes de ovos de Anagasta kuehniella tratados, contendo o parasitóide nas diferentes fases imaturas ${ }^{(1)}$.

\begin{tabular}{|c|c|c|c|}
\hline Tratamento & Ovo-larva & Pré-pupa & Pupa \\
\hline & \multicolumn{3}{|c|}{ Emergência } \\
\hline Acetamipride & $95,3 \pm 0,79 \mathrm{aA}$ & $91,4 \pm 1,60 \mathrm{aB}$ & $94,9 \pm 0,86 \mathrm{aA}$ \\
\hline Clorfenapir & $95,5 \pm 1,48 \mathrm{aA}$ & $96,3 \pm 1,36 \mathrm{aA}$ & $94,5 \pm 1,83 \mathrm{aA}$ \\
\hline Imidaclopride & $93,9 \pm 1,37 \mathrm{aA}$ & $97,5 \pm 0,76 \mathrm{aA}$ & $96,4 \pm 0,93 \mathrm{aA}$ \\
\hline Tiaclopride & $93,5 \pm 1,26 \mathrm{aA}$ & $95,0 \pm 0,88 \mathrm{aA}$ & $92,6 \pm 1,28 \mathrm{aA}$ \\
\hline Tiametoxam & $95,5 \pm 0,90 \mathrm{aA}$ & $91,2 \pm 0,76 \mathrm{aB}$ & $95,0 \pm 0,95 \mathrm{aA}$ \\
\hline Testemunha & $90,9 \pm 3,92 \mathrm{aA}$ & $92,6 \pm 4,51 \mathrm{aB}$ & $95,1 \pm 1,04 \mathrm{aA}$ \\
\hline \multirow[t]{2}{*}{ Média } & 94,1 & 94,0 & 94,8 \\
\hline & \multicolumn{3}{|c|}{ Adultos deformados } \\
\hline Acetamipride & $0,9 \pm 0,26 \mathrm{aA}$ & $0,3 \pm 0,17 \mathrm{aA}$ & $0,2 \pm 0,07 \mathrm{aA}$ \\
\hline Clorfenapir & $0,0 \pm 0,00 \mathrm{aA}$ & $0,0 \pm 0,00 \mathrm{aA}$ & $0,7 \pm 0,33 \mathrm{aA}$ \\
\hline Imidaclopride & $0,4 \pm 0,18 \mathrm{aA}$ & $0,0 \pm 0,00 \mathrm{aA}$ & $0,4 \pm 0,22 \mathrm{aA}$ \\
\hline Tiaclopride & $0,4 \pm 0,18 \mathrm{aA}$ & $0,6 \pm 0,20 \mathrm{aA}$ & $0,5 \pm 0,23 \mathrm{aA}$ \\
\hline Tiametoxam & $0,9 \pm 0,42 \mathrm{aA}$ & $0,9 \pm 0,41 \mathrm{aB}$ & $0,5 \pm 0,25 \mathrm{aA}$ \\
\hline Testemunha & $1,2 \pm 0,51 \mathrm{aA}$ & $1,4 \pm 0,54 \mathrm{aB}$ & $1,1 \pm 0,64 \mathrm{aA}$ \\
\hline Média & 0,6 & 0,5 & 0,6 \\
\hline \multicolumn{4}{|c|}{$\begin{array}{l}\text { (1) Médias seguidas pela mesma letra, minúscula na linha e maiúscula na } \\
\text { coluna, não diferem entre si pelo teste de Scott-Knott }(\mathrm{P}<0,05) \text {; dados } \\
\text { transformados para o arc sen da raiz quadrada de } \mathrm{x} / 100 \text {; CV (\%): 6,4 } \\
\text { (emergência) e } 42,5 \text { (adultos deformados). }\end{array}$} \\
\hline
\end{tabular}

respectivamente. Entretanto, não foram observadas diferenças entre os estágios de desenvolvimento desse parasitóide, em relação à porcentagem de indivíduos deformados, inclusive nos tratamentos testemunha e tiametoxam (Tabela 4). As porcentagens observadas de deformação de indivíduos da geração $\mathrm{F}_{2}$ (Tabela 4), nas diferentes épocas de aplicação, são consideradas baixas, segundo taxas de controle de qualidade, pois segundo Haji et al. (1998b), é considerada normal a ocorrência de até $2 \%$ de indivíduos atípicos em criações massais de Trichogramma spp.

\section{Conclusões}

1. Os produtos clorfenapir e imidaclopride reduzem a porcentagem de emergência de $T$. pretiosum da geração $F_{1}$, quando aplicados sobre esse parasitóide nas suas diversas fases imaturas.

2. Nenhum dos compostos avaliados provoca alterações na proporção de fêmeas e machos de $T$. pretiosum da geração $F_{1}$.

3. Clorfenapir reduz a taxa de parasitismo de $T$. pretiosum da geração $\mathrm{F}_{1}$, independentemente da fase de desenvolvimento desse parasitóide.

4. Acetamipride e tiametoxam são, em geral, inofensivos a $T$. pretiosum.

\section{Agradecimentos}

À Capes e ao CNPq, pela concessão de bolsas a Alexandre Pinho Moura.

\section{Referências}

BLEICHER, E.; PARRA, J.R.P. Espécies de Trichogramma parasitóides de Alabama argillacea III. Determinação das exigências térmicas de três populações. Pesquisa Agropecuária Brasileira, v.25, p.215-219, 1990.

BOTELHO, P.S.M. Eficiência de Trichogramma em campo. In: PARRA, J.R.P.; ZUCCHI, R.A. (Ed.). Trichogramma e o controle biológico aplicado. Piracicaba: FEALQ, 1997. p.303-318.

BRUNNER, J.F.; DUNLEY, J.E.; DOERR, M.D.; BEERS, E.H. Effect of pesticides on Colpoclypeus florus (Hymenoptera: Eulophidae) and Trichogramma platneri (Hymenoptera: Trichogrammatidae), parasitoids of leafrollers in Washington. Journal of Economic Entomology, v.94, p.1075-1084, 2001.

CARVALHO, G.A.; MIRANDA, J.C.; VILELA, F.Z.; MOURA, A.P.; MORAES, J.C. Impacto de inseticidas sobre vespas predadoras e parasitóides e sua eficiência no controle de Leucoptera coffeella (Guérin-Mèneville \& Perrottet, 1842) (Lepidoptera: Lyonetiidae). Arquivos do Instituto Biológico, v.71, p.63-70, 2004. 
CARVALHO, G.A.; PARRA, J.R.P.; BAPTISTA, G.C. Seletividade de alguns produtos fitossanitários a duas linhagens de Trichogramma pretiosum Riley, 1879 (Hymenoptera: Trichogrammatidae). Ciência e Agrotecnologia, v.25, p.583-591, 2001.

CARVALHO, G.A.; REIS, P.R.; ROCHA, L.C.D.; MORAES, J.C.; FUINI, L.C.; ECOLE, C.C. Side-effects of insecticides used in tomato fields on Trichogramma pretiosum (Hymenoptera, Trichogrammatidae). Acta Scientiarum, v.25, p.275-279, 2003.

CÔNSOLI, F.L.; BOTELHO, P.S.M.; PARRA, J.R.P. Selectivity of insecticides to egg parasitoid Trichogramma galloi Zucchi, 1988, (Hymenoptera: Trichogrammatidae). Journal of Applied Entomology, v.125, p.37-43, 2001.

CROFT, B.A. Arthropod biological control agents and pesticides. New York: Wiley-Interscience, 1990. 723p.

GUIFEN, Z.; HIRAI, K. Effects of insecticides on developmental stages of Trichogramma japonicum in the laboratory. Proceedings of the Kanto Tosan Plant Protection Society, v.44, p.197-200, 1997.

HAJI, F.N.P.; ALENCAR, T.A.; PREZOTTI, L. Principais pragas do tomateiro e alternativas de controle. Petrolina: EmbrapaCPATSA, 1998a. 51p.

HAJI, F.N.P.; JIMENEZ VELASQUEZ, J.; BLEICHER, E.; ALENCAR, J.A.; HAJI, A.T.; DINIZ, R.S. Tecnologia de produção massal de Trichogramma spp. Petrolina: Embrapa-CPATSA, 1998 b. $24 \mathrm{p}$

HASSAN, S.A.; HAFEZ, M.B.; DEGRANDE, P.E.; HIRAI, K. The side-effects of pesticides on the egg parasitoid Trichogramma cacoeciae Marchal (Hym., Trichogrammatidae), acute dose-response and persistence tests. Journal of Applied Entomology, v.122, p.569-573, 1998.

McLEOD, P.; DIAZ, F.J.; JOHNSON, D.T. Toxicity, persistence and efficacy of spinosad, chlorfenapyr, and thiamethoxam on eggplant when applied against the eggplant flea beetle (Coleoptera: Chrysomelidae). Journal of Economic Entomology, v.95, p.331335, 2002.

NOVOZHILOV, K.V.; KAMENKOVA, K.V.; SMIRNOVA, I.M. Development of the parasite Trissolcus grandis Thoms. (Hymenoptera, Scelionidae) where organophosphorus insecticides are in use against Eurygaster integriceps Put. (Hemiptera, Scutelleridae). Entomologicheskoe Obozrenie, v.52, p.20-28, 1973.

O'BRIEN, P.J.; ELZE, G.W.; VINSON, S.B. Toxicity of azinphosmethyl and chlordimeform to parasitoid Bracon mellitor
(Hymenoptera: Braconidae): lethal and reproductive effects. Environmental Entomology, v.14, p.891-894, 1985.

PARRA, J.R.P. Técnicas de criação de Anagasta kuehniella, hospedeiro alternativo para produção de Trichogramma. In: PARRA, J.R.P.; ZUCCHI, R.A. (Ed.). Trichogramma e o controle biológico aplicado. Piracicaba: FEALQ, 1997. p.121-150.

SCHULD, M.; SCHMUCK, R. Auswirkungen des chloronicotinylinsektizids imidacloprid auf die parasitoidenfauna in obstanlagen. Mitteilungen der Deutschen Gesellschaft für Allgemeine und Angewandte Entomologie, v.11, p.265-270, 1997.

SCHULD, M.; SCHMUCK, R. Effects of thiacloprid, a new chloronicotinil insecticide, on the egg parasitoid Trichogramma cacoeciae. Ecotoxicology, v.9, p.197-205, 2000.

SCOTT, A.J.; KNOTT, M.A. A cluster analysis method for grouping means in the analysis of variance. Biometrics, v.30, p.507-512, 1974.

STEIN, C.P.; PARRA, J.R.P. Uso da radiação ultra-violeta para inviabilizar ovos de Anagasta kuehniella (Zeller, 1879) visando estudos com Trichogramma spp. Anais da Sociedade Entomológica do Brasil, v.16, p.163-169, 1987.

STERK, G.; HASSAN, S.A.; BAILLOD, M.; BAKKER, F.; BIGLER, F.; BLÜMEL, S.; BOGENSCHÜTZ, H.; BOLLER, E.; BROMAND, B.; BRUN, J.; CALIS, J.N.M.; COREMANSPELSENEER, J.; DUSO, C.; GARRIDO, A.; GROVE, A.; HEIMBACH, U.; HOKKANEN, H.; JACAS, J.; LEWIS, G.; MORETH, L.; POLGAR, L.; ROVERSTI, L.; SAMSØEPETERSEN, L.; SAUPHANOR, B.; SCHAUB, L.; STÄUBLI, A.; TUSET, J.J.; VAINIO, A.; VAN DE VEIRE, M.; VIGGIANI, G.; VIÑUELA, E.; VOGT, H. Results of the seventh joint pesticide testing programme carried out by the IOBC/WPRS-Working Group 'Pesticides and Beneficial Organisms'. BioControl, v.44, p.99-117, 1999.

TAKAHASHI, H.; TAKAKUSA, N.; SUZUKI, J.; KISHIMITO, T. Development of a new insecticide, acetamiprid. Journal of Pesticide Science, v.23, p.193-200, 1998.

TREACY, M.; MILLER, T.; BLACK, B.; GARD, I.; HUNT, D.; HOLLINGWORTH, M.R. Uncoupling activity and pesticidal properties of pyrroles. Biochemical Society Transactions, v.22, p.244-247, 1994.

YAMAMOTO, I.; YABUTA, G.; TOMIZAWA, M.; SAITO, T.; MIYAMOTO, T.; KAGABU, S. Molecular mechanism for selective toxicity of nicotinoids and neonicotinoids. Journal of Pesticide Science, v.20, p.33-40, 1995. 